\title{
"Ojo clínico" y evidencia científica
}

\author{
Francisco Gudiol Munté
}

Facultad de Medicina. Universidad de Barcelona. Jefe de Servicio de Enfermedades Infecciosas Hospital Universitario de Bellvitge.

\section{INTRODUCCIÓN}

En nuestra profesión, el ojo clínico puede definirse como el don que poseen algunos médicos para realizar diagnósticos rápidos y certeros. Este término, antaño popular, ha caído en desuso y en la actualidad se utiliza con mayor frecuencia en ambientes no médicos, para resaltar la habilidad de anticipar acontecimientos o de calibrar a simple vista aspectos ocultos de la naturaleza humana.

Por el contrario, el concepto evidencia científica es claramente actual. Aplicado a la práctica de la medicina, no solo es actual, sino que además está de moda. La medicina basada en la evidencia se define como la utilización consciente, explícita y juiciosa de la mejor evidencia científica disponible para tomar decisiones sobre el cuidado de pacientes individuales. El sentido común indica que es mejor tener ojo clínico que estar poco dotado para orientar con agilidad el diagnóstico de los pacientes. Sin embargo, esta cualidad ha sido denostada con frecuencia (en general por profesionales alejados de la práctica clínica), siendo equiparada a la arbitrariedad en la toma de decisiones. En este sentido se ha afirmado que utilizar el ojo clínico tiende a deteriorar la calidad de la atención médica, ejerciendo un efecto perjudicial sobre el crédito profesional.

La medicina basada en la evidencia tiene también sus detractores (en general profesionales alejados de la moderna tecnología) que la acusan de limitar la libertad de actuación de los clínicos, de un exceso de rigidez y esquematismo y de sometimiento al pensamiento dominante.

Sin embargo, en opinión de muchos, los dos conceptos no deberían ser antagónicos, sino complementarios.

\section{LA PERICIA CLÍNICA}

La práctica clínica es el proceso de la actuación médica relacionada con la atención a la salud del paciente. Sus componentes son la información clínica obtenida, las percepciones, los razonamientos, los juicios, los procedimientos utilizados, las decisiones y las intervenciones que se aplican.

El análisis inmediato de tal definición nos indica que nos hallamos ante un fenómeno complejo, inexacto y difícilmente reproducible.

Según los clásicos, la práctica de la medicina se fundamenta en la combinación de la ciencia y el arte. Pocos dudan de la importancia de la ciencia, pero el arte de la medicina es también una parte relevante de la ecuación, si lo entendemos como una mezcla de conocimientos, intuición y buen criterio, ingredientes necesarios para la correcta aplicación clínica de la tecnología científica.

En mi opinión, el término que mejor define el grado de capacidad de un médico para el ejercicio profesional es el de pericia clínica, que los anglosajones denominan "expertise". La pericia clínica (mucho mejor que ojo clínico) permite al médico establecer un buen juicio clínico, entendido como un proceso de toma de decisiones en ausencia de leyes o reglas explícitas. El poder deductivo asociado a la pericia clínica no se fundamenta solo en los años de experiencia, sino también en la observación y el estudio continuados, el rigor en la obtención de datos y el sentido común.

El procedimiento clínico abarca desde la primera entrevista con el paciente hasta la decisión de un diagnóstico tentativo. Muchas enfermedades comparten signos y síntomas; a su vez, muchas manifestaciones clínicas no necesariamente representan 
enfermedad. La entrevista, el examen físico y los exámenes complementarios reducen el enorme conjunto de posibilidades a un pequeño número de diagnósticos diferenciales. Si lleváramos el proceso a un gráfico, la figura podría parecerse a un embudo; a medida que el proceso diagnóstico avanza, el universo cognoscitivo necesario se reduce. En el extremo más estrecho del embudo es donde la aplicación de la informática (bases de datos, sistemas expertos) pueden ser de mayor utilidad, pero la pericia clínica es fundamental para llegar hasta él.

La expresión máxima de la pericia clínica se sitúa en el último de los cinco escalones del nivel de habilidades de Dreyfus (novel, principiante avanzado, competente, diestro y experto), caracterizado por la captación intuitiva y profunda de las situaciones, por la no utilización de reglas, normas ni guías, por el inicio de procesos analíticos solo ante situaciones nuevas y por una visión rápida de aquello que es posible.

\section{LA VARIABILIDAD DE LA PRÁCTICA MÉDICA}

La práctica médica ha sido y sigue siendo heterogénea, no sólo porque como se ha dicho repetidamente "no existen enfermedades sino enfermos" sino también porque existe un alto grado de variabilidad en el nivel de pericia clínica entre facultativos. Esta variabilidad, que afecta a todas las fases del razonamiento clínico y de la toma de decisiones (Tabla 1), se ha hecho más patente en el transcurso de las últimas

\section{Tabla 1. Fases del razonamiento clínico y de la toma de decisiones}

1) Estudio clínico (anamnesis y exploración física)

2) Práctica de las pruebas diagnósticas

3) Integración de los hallazgos anteriores

4) Ponderación de riesgos y beneficios

5) Determinación de las preferencias del paciente

6) Desarrollo de un plan terapéutico

\section{Tabla 2. Variabilidad de la práctica médica}

1) Características de paciente

2) Características de la población

3) Características del sistema sanitario

4) Características personales del médico

5) Calidad de la evidencia científica utilizada décadas. Los enormes avances en el campo de la tecnología diagnóstica y en la prevención y tratamiento de las enfermedades han hecho que sea imposible para cualquiera de nosotros mantenerse al día en el conocimiento de tales avances, en su interpretación adecuada y en la conveniencia de su aplicación a nuestros pacientes, utilizando los recursos convencionales. Los conocimientos no pueden mantenerse actualizados mediante el uso exclusivo de libros de texto, que pronto quedan obsoletos, y de artículos de opinión escritos por expertos, a menudo de objetividad poco contrastada. La enorme cantidad de información científica generada por la investigación biomédica, en franca expansión, se halla a nuestro alcance a través de los artículos publicados en las revistas médicas. Sin embargo, su cantidad es tan enorme que su revisión continuada (incluso limitada a la propia especialidad) desborda con mucho las posibilidades logísticas de un médico en ejercicio. Asimismo, es obvio que la información científica que se publica es de muy distinta calidad, por lo que su asimilación "acrítica" puede ser en ocasiones más contraproducente que útil. El crecimiento exponencial de la literatura médica combinado con el fácil acceso a la información gracias a los ordenadores personales y a internet resulta en una avalancha de información que obliga al médico responsable a utilizar recursos informáticos que le permitan identificar la información verdaderamente relevante para su práctica diaria. En este contexto, éste necesita un instrumento que le permita separar rápidamente el heno de la paja, puesto que con el ritmo actual de realización de proyectos de investigación y producción de ensayos clínicos la cuestión no es sólo mantenerse informado sino también cuanta de la evidencia disponible es realmente útil y cuanta se aplica realmente en el manejo diario de los pacientes.

En consecuencia, entre las distintas razones que pueden explicar la variabilidad de la práctica médica (Tabla 2), tanto a nivel individual como poblacional o relacionadas con las características del sistema sanitario, las diferencias en la cantidad y calidad de la evidencia científica utilizada por los profesionales se consideran en la actualidad de gran importancia para entender las diferencias existentes en la calidad asistencial prestada por los médicos.

\section{LA MEDICINA BASADA EN LA EVIDENCIA}

La aparición y posterior desarrollo de la medicina basada en la evidencia se fundamenta en la creencia de que actualmente la información derivada de la 


\section{Tabla 3. El proceso de la medicina basada en la evidencia}
1) Formulación de la pregunta clínica
2) Búsqueda bibliográfica (bases de datos)
3) Valoración de validez y aplicabilidad
4) Aplicación de la evidencia obtenida
5) Evaluación clínica de los resultados

experiencia clínica y la intuición, aunque necesaria, puede ser insuficiente para establecer decisiones adecuadas si no se basa en la información sólida proporcionada por las observaciones sistemáticas. Para evaluar rigurosamente la metodología con la que se han obtenido las pruebas científicas en las que se sustentan las decisiones, es preciso conocer ciertas reglas y aplicar la metodología pertinente. La medicina basada en la evidencia incorpora al arsenal de conocimientos científicos y habilidades básicas para el desempeño de la profesión médica, la destreza en el uso de una serie de conocimientos sencillos de estadística, diseño de investigaciones y epidemiología clínica. Así pues, los médicos deben adquirir la responsabilidad de evaluar de forma crítica e independiente la credibilidad de las evidencias y de las opiniones enunciadas.

La evidencia obtenida debe integrarse con la pericia clínica individual y las expectativas, preferencias $\mathrm{y}$ deseos del paciente. El proceso de la medicina basada en la evidencia puede resumirse en cinco puntos, que se enuncian en la Tabla 3. La actividad clínica genera interrogantes acerca de la etiología de una determinada dolencia, la utilidad de una prueba diagnóstica, el pronóstico de la enfermedad o los efectos de una posible terapia, lo que lleva al planteamiento de una pregunta clínica, punto inicial del proceso. A continuación se realiza la búsqueda bibliográfica correspondiente, evaluando posteriormente su validez y aplicabilidad. La aplicación de la evidencia considerada debe realizarse siempre a la luz del mejor juicio clínico. El punto final del proceso es la evaluación clínica del resultado obtenido.

La práctica y aplicación de la medicina basada en la evidencia no son sencillas. Es esencial formular con precisión y sentido clínico la pregunta clínica que inicia el proceso, así como seleccionar los tipos de estudio más adecuados en función de la pregunta, conociendo los grados de evidencia de cada uno. Debemos asimismo saber buscar la información en las fuentes idóneas, ya sean bases de datos con filtro de calidad o en revistas secundarias que nos ofrecen información ya revisada y catalogada desde el punto de vista de la evidencia a partir de artículos de metodología sólida.

Finalmente, en la medida que la medicina basada en la evidencia se considere un elemento clave de la práctica médica, sus fundamentos deberían aplicarse también a la docencia de la medicina. De esta forma los estudiantes recibirían probablemente una información más objetiva y homogénea y se graduarían conociendo ya la importancia de la evidencia científica y estando familiarizados con la forma de obtenerla. Sin embargo, aunque existe una fuerte corriente a favor de la aplicación sistemática de esta metodología en las universidades, se trata todavía de una realidad muy lejana.

\section{CONCLUSIONES}

1) El "ojo clínico" (que hemos bautizado como pericia clínica) y la evidencia científica no son conceptos enfrentados sino complementarios

2) La pericia clínica es difícil de obtener y sus valores deberían salvaguardarse

3) La práctica de la medicina basada en la evidencia requiere la integración de la mejor evidencia científica, la pericia clínica individual y los valores de los pacientes

4) La sistemática de trabajo propuesta por la medicina basada en la evidencia debe ser adoptada como un elemento básico de la práctica médica

5) La metodología de la medicina basada en la evidencia debe aplicarse también a la docencia de la medicina

\section{BIBLIOGRAFÍA}

1. Flyvbjerg B. Sustaining Non-rationalized Practices: BodyMind, Power and Situational Ethics: An Interview with Hubert and Stuard Dreyfus. Institute of Development and Planing, University of Aalborg, 1992

2. Evidence-based medicine working group. Evidence-based medicine: a new approach to teaching the practice of medicine. JAMA 1988; 259: 3.277-3.280

3. Sackett DL, Rosenberg WMC, Gray JA, Haynes RB, Richardson WS. Evidence-based medicine: what itis and what it isn't. BMJ 1996; 312: 71-72

4. Van Der Vleuten CPM, Dolmans DHJM and Scherpbier AJJA. The need for evidence in education. Medical Teacher 2000; 22 (3): $246-250$ 